\title{
An Overview of Service Composition in Service Oriented Architecture
}

\author{
Faten hamad ${ }^{1}$ \\ ${ }^{1}$ School of Educational sciences, The university of Jordan, Amman, Jordan \\ Correspondence: Faten Hamad, School of Educational sciences, The University of Jordan, Amman, Jordan. \\ E-mail: f.hamad@ju.edu.jo
}

Received: June 27, 2018

Accepted: July 6, 2018

Online Published: July 28, 2018

doi:10.5539/mas.v12n8p172

URL: https://doi.org/10.5539/mas.v12n8p172

\begin{abstract}
Service oriented architecture (SOA) is a form of software design in which application component supply services to other components through a network communication protocol, it has many services that can transfer small data with communication channels or additional services which bring into a relationship that ensure efficiency of service activities, SOA simplify the structure of loosely coupled applicable applications and enable contribution for enterprise working of services together. In order to assure the effectiveness of Service oriented architecture we have to confirm service composition which is the collection of services together in order to perform a specific function which can be used in service oriented architecture. In this paper we proposed a Service composition in SOA, it is present service composition with various techniques used for composing services and provided a comparison between them.
\end{abstract}

Keywords: service composition, SOA, service oriented architecture

\section{Introduction}

The development of Internet technology has led to the creation and application of service oriented architecture (SOA) software systems based on application functions in the form of program services to which Access to the Internet or intranet environment of non-depending on the location in the global computer network .Principles of Existing service-oriented-architects do not take into account the functions performed by the services from the features of the model of the problematic and tasks to be solved, the tasks optimal selection of services from a variety of available and their dynamic adaptation to the specific needs, which can lead to the adoption of effective solutions for organizing information processes (Kim \& Hwa, 2016).

automation the SOA optimization problem solving to find a solution for it request an efficient methods by software engineers like using search-based software engineering (SBSE) which is a method that perform the optimization techniques of meta-heuristic search-based, we can use Genetic algorithms based on heuristic search to solve optimization problems (Lifang, Wenjian and Hang, 2017).

Service composition represent transferring user functional and nonfunctional requirement to execution plan of service composition as Service Level Agreement (SLA) to detect the services that achieve those requirements, Service Level Agreement can be structure, scenario or optimal execution plan. General directions to companies and individual systems of getting help from external sources tasks build a requirement for automated service composition. a lot of service composition approaches have been presented before, the utilize of AI Planning techniques was firstly introduced lead to two-staged composition, then different approaches presented like workflow-based approach and template-based approach, and more lately service composition approaches in obvious of semantic match emergence service composition development and implementing of Quality of Service (QoS) requirements (Huf, Salvadori and Siqueira, 2017).

This paper is structured as follows: Section II introduces the existing service composition surveys. Section III discusses the service composition techniques. Finally, section IV presents our conclusions

\section{Related Terminology}

In this section we will illustrate the web service composition, service oriented architecture, dynamic service composition and finally the service composition framework each one of them as a subsection. 


\subsection{Web Services Composition}

A Web service is a network technology that provides program communication (between different applications) based on Web standards. Web-services allow you to access from one application to another and at the same time perform certain functions. The application solution can be a provider of web services or a consumer of web services published by other providers. It is a network technology that provides program communication (between different applications) based on Web standards. Web-services allow you to access from one application to another and at the same time perform certain functions. The Web service has a programming interface, represented in the WSDL (Web Services Description Language) format, a language for describing and accessing Web services, based on the XML language, Designed for unified presentation of external web interfaces (Alessandra et al., 2017) (Hamad \& Adwan, 2018).

FEKIH et al. proposed a new approach based on local consistency build up methods to enhance the skyline approach and to reach the user requirements. The Harmony Search algorithm adopted to catch a near-optimal composition. The experimental evaluation of the proposed approach is reduced the size of classic Skyline in a reasonable time computation (fekih, mtibaa and bouamama, 2017). Also, Elli Rapti et al., adopted the artificial potential fields that can be mapped to the service composition problem in Internet of Thing networks to achieve global equilibrium through local interactions (Rapti et al., 2017). Also, Elli Rapti et al. adopted the artificial potential fields that can be mapped to the service composition problem in Internet of Thing networks to achieve global equilibrium through local interactions (Rapti et al., 2017). Moreover, Yuqian Lu and Xun Xu (217) examines knowledge-based service composition and adaptive resource planning in dynamic cloud environment by developing a systematic and easy to be implement service composition mechanism. They used system utilizes distributed knowledge for intelligent service composition and adaptive resource planning.

Kamath et al. (2013) Recommend a framework that employ A base up methodology to constructing semantic Web services starting with existing service descriptions on the Web, Subsequently decreasing those duration of the time Furthermore cosset included in substantial scale manual annotation.

Figure 2. Shows the web service composition Workflow techniques which classified into orchestration and choreography. In orchestration model, the engaged web services are under control of a single central process (another web service). In choreography model, it does not depend on a central point, all web services should participate. Moreover, in the choreography has to know exactly when to become active and with whom to interoperate (Nagamouttou et al., 2015)

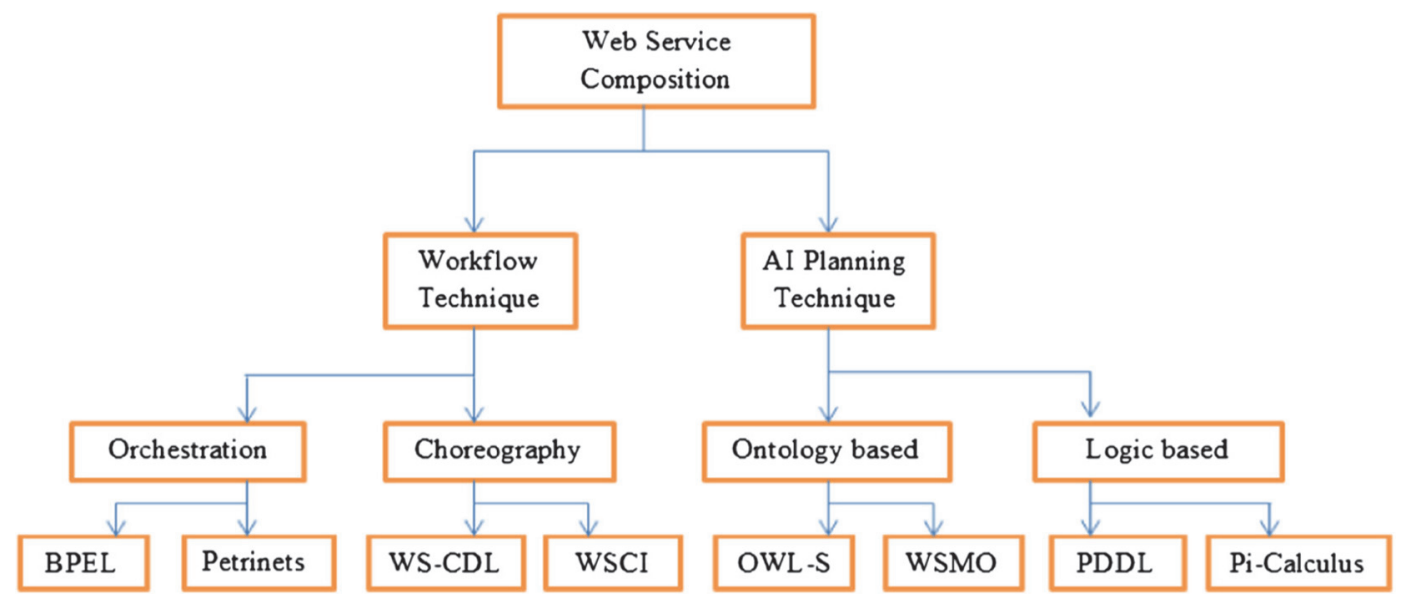

Figure 1. The flow of web service composition

\subsection{Dynamic Service Composition}

it is the mechanism in which Application services at runtime is connected with a lot of data services, state of being interconnected is deployed dynamically based on the characteristics of database management, for effective data accessing a lot of cloud databases runtime is being provision by connectivity of Data service dynamic and Application service (Li et al., 2017) (Hudaib \& Fakhouri, 2016).

Wan et al., proposed a novel pattern built element service framework that merge outlined pattern What's more design implementation methodologies to prepare composited services. The pattern may be formalized Likewise 
an uncommon chart similitude matching issue Furthermore an algorithm may be outlined will figure those similitude about these services creation results (Wang, Zhitao Wan \& Ping, 2017).

Zhang et al. Recommend an algorithm MR-IDPSO (MapReduce according to progressed discrete particle swarm Optimization), which makes utilization of those moved forward discrete particle swarm optimization (PSO) for those Map Reduce should solved extensive scale dynamic service composition. Analyses indicate that our algorithm outperforms the parallel genetic algorithm as far as result of effective vast scale dynamic service composition (Pal, Anshuk Dubey and Sanjukta, 2017).

\section{Service Composition Framework}

We looked at how to maximize the potential of an SOA solution by identifying business closely connected services framework which is being designed based on work requirements and service contract that the services solution must accomplish, a common framework for service composition system is being listed bellow in figure 1 , this framework proposed the fundamentals of service composition methods symmetry and variations (Pal, Anshuk Dubey \& Sanjukta, 2017).

The Service composition framework contains the following components: translator, evaluator, process generator, and execution engine and service repository. And have two main participants' service requester and service provider, service requester firsthand participants who suggest the service in order to be used through the framework which provide external specification to the translator (Pal, Anshuk Dubey \& Sanjukta, 2017).

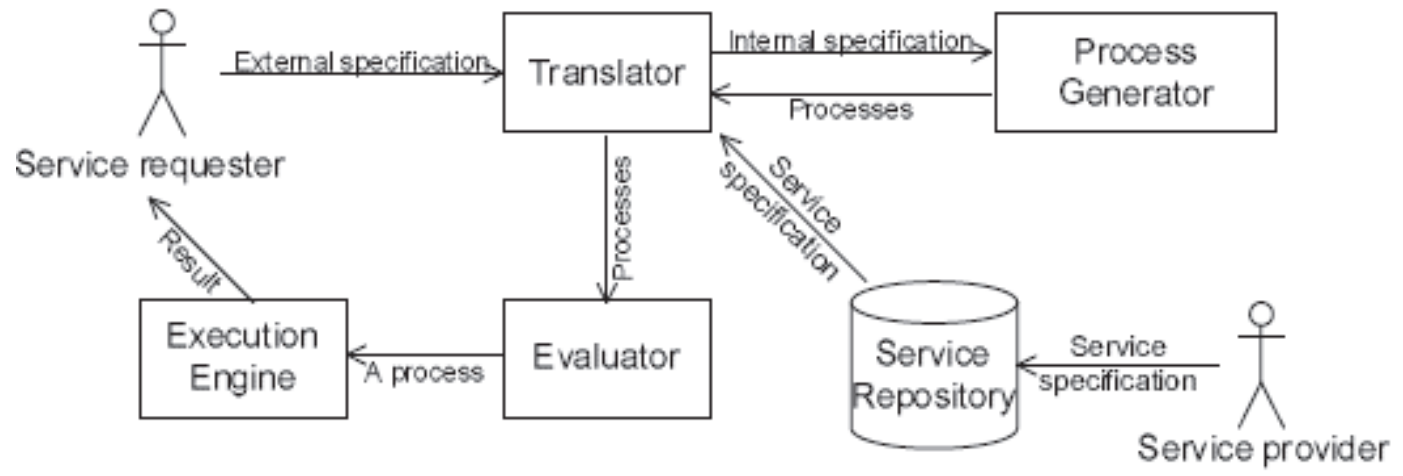

Figure 2. The framework of the service composition system

The service requesters expand service provider's services and data. The translator is a connecter between service provider and process generator to make the language understandable for process generator by translating external specification language used by service requester to the language used by process generator. The execution engine executes the process and plan of data from the evaluator and sends the output to the service requester. For every request translator sends internal specification to process generator. For each request, the process generator produce a plan which control the existing service repository services in order to accomplish the request. If there are many plans the evaluator evaluates all plans and give the leading service for processing (Baldin, Shu Huang \& Ilya, 2016)

Service compositions can be classified into primitive and complex variations. In early service-oriented solutions, simple logic was generally implemented via point-to-point exchanges or primitive compositions. As the surrounding technology matured, complex compositions became more common see figure 4 .

Much of the service-orientation design paradigm revolves around preparing services for effective participation in numerous complex compositions. So much so that the Service Compensability design principle exits, dedicated solely to ensuring that services are designed in support of repeatable composition. 


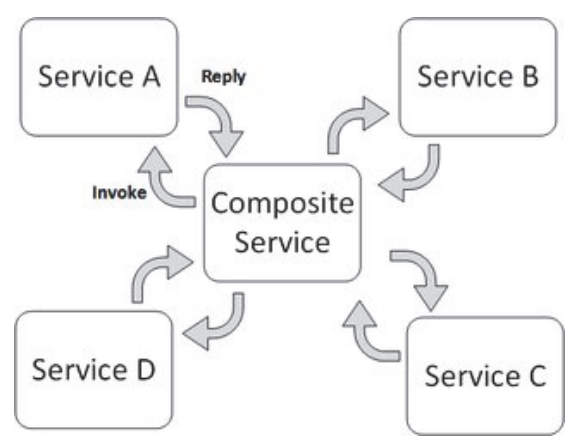

Figure 4. Service composition communication

\section{Service-Orientation and the Concept of "Application"}

Service-orientation places an unprecedented emphasis on reuse. By establishing a service inventory with a high percentage of reusable and agnostic services, we are now positioning those services as the primary (or only) means by which the solution logic they represent can and should be accessed.

As a result, a very deliberate move away from the silos in which applications previously existed. Because we want to share reusable logic whenever possible, we automate existing, new, and augmented business processes through service composition. This results in a shift where more and more business requirements are fulfilled not by building or extending applications, but by simply composing existing services into new composition configurations (Al-Sayyed et al., 2017).

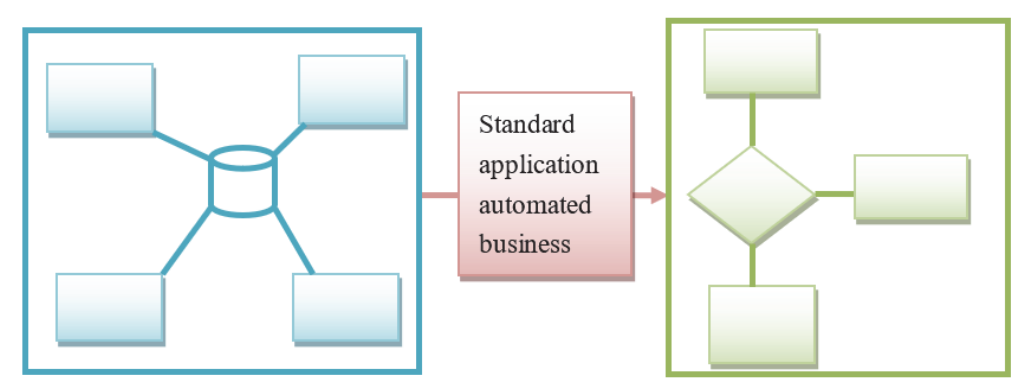

Figure 5. The traditional application, delivered to automate specific business process logic

When compositions become more common, the traditional concept of an application or a system or a solution actually begins to fade, along with the silos that contain them. Applications no longer consist of self-contained bodies of programming logic responsible automating a specific set of tasks. What was an application is now just another service composition. And, it's a composition made up of services that very likely participate in other compositions (Al Khattab et al., 2015).

Table 1. Service composition approaches according to each researcher and the techniques used

\begin{tabular}{lll}
\hline $\begin{array}{l}\text { Composition } \\
\text { approaches }\end{array}$ & Researcher & techniques \\
\hline Meteror-s & $\begin{array}{l}\text { Aggarwal } \\
\text { al. (2015) }\end{array}$ & $\begin{array}{l}\text { Present demand Web Service Composition composition device in } \\
\text { METEOR-S, which permits those transform designers on Web benefits with a } \\
\text { conceptual process, In view of business and procedure imperatives What's } \\
\text { more produce an executable procedure. } \\
\text { Approach may be to decrease a great part of the Service Composition issue will } \\
\text { a demand fulfillment issue. It utilization A multi-phase methodology to } \\
\text { demand dissection. This worth of effort might have been done Similarly as and } \\
\text { only the METEOR-S framework, which plans on backing the complete } \\
\text { lifecycle from claiming semantic Web forms. }\end{array}$ \\
& &
\end{tabular}

\section{Sword}

Asmethal et Recommended Web service framework is used to give services in an ideal way. 
al. (2012) In the suggested structural engineering five levels are utilized. Benefits need aid made independently with those help of a toolkit thus those seniors could select their service effectively. Web service Adapters need aid utilized for conveying for a few Web services. It may be necessary for build the composite benefits manually. This will be particularly vital on account of composite service formation may be not so much a one-time exertion.

\section{Wsmo \\ Template \\ based \\ composition}

Siddiqui

al. (2012)

et Pro mposition methodology the middle of two phases. Format built composition employments design-time will request those placeholders Furthermore to join them, Furthermore utilization run-time will fill the placeholders for those real execution.

Shop2

Parsia et al.

Star WSCop

Web service

\section{SECSE}

QoS (2013)

Bartalos et al. (2011)

Wang et al. Identify the challenges around service compositions: evolving execution (2011)

KASSMI et Recommend in compositions for security prerequisites. It comprises on al. (2016) demonstrating some security necessities utilizing FSM (Finite state Machine) Furthermore their mix rapidly with communicated utilitarian prerequisites. On accept our formalization What's more mix approach clinched alongside web service composition, we bring utilized model checking apparatus UPPAAL.

developed SHOP2 usage on arrange for What's more over the translated domain, et cetera executes those coming about compositions. In the methodology about characterizing the interpretation Furthermore building those framework, present approach, the organizer constantly executes yield handling activities Concerning illustration its compositions. Same time this is fine to huge numbers situations, it might not continuously make suitable.

Suppose that service composition to be examined likewise from programming building purpose from claiming perspective. A technique must make produced describing the methodology about Web service design, semantic annotation, maintenance, and the use about Web service composition. Devices supporting these assignments must make Gave. Currently, it will be not clear for which path is it useful with misuse service creation. We don't recognize On it to be connected best to other domains, if we by any means might successfully utilization state funded services or must depend looking into private ones main. Exploration consideration if make committed should discover An executioner provision demonstrating to those reductions and limits about service composition On act. suppliers. With face of these challenges, they configuration and execute An self-adaptive Also context-aware service composition system, which might tackle setting occasions Also settle on changing adaptations as stated by user-defined customize strategies.

Paik et al. Suggested adjustable transaction What's more QoS-aware service Choice (2009) contexts, self-adaptation during runtime, and the workload about service approach under five client custom settings In light of hereditary calculation (GA) on deliver over worries. QoS-awareness encouraged Eventually Tom's perusing multi-objective QoS criteria Also ga is utilized to multivariate streamlining. We led An careful evaluation, Also it reveals to suggested technique viably Also effectively scope the worldwide ideal of the generally determination criteria.

\section{Service Compositions and Technology Architecture}

Because applications have existed for as long as IT, when technology architecture as a profession and perspective within the enterprise came about, it made perfect sense to have separate architectural views dedicated to individual applications, integrated applications, and the enterprise as a whole.

When standardizing on service-orientation, the manner in which we document technology architecture is also in 
for a change. The enterprise-level perspective becomes predominant as it represents a master view of the service inventory. It can still encompass the traditional parts of a formal architecture, including conceptual views, physical views, and supporting technologies and governance platforms - but all these views are likely to now become associated with the service inventory (Hudaib et al, 2016).

A new type of technical specification that gains prominence in service-oriented enterprise initiatives is the service composition architecture. Even though we talk about the simplicity of combining services into new composition configurations on demand, it is by no means an easy process. It is a design exercise that requires the detailed documentation of the planned composition architecture.

For example, each service needs to be assessed as to its competency to fulfill its role as a composition member, and foreseeable service activity scenarios need to be mapped out. Message designs, messaging routes, exception handling, cross-service transactions, policies, and many more considerations go into making a composition capable of automating its designated business process.

\section{Conclusion}

The number of publicly accessible service composition techniques and approaches are growing, so we see a fast improvement in service composition technologies this survey presented based on different techniques like Automated based service composition, Process algebra base service composition, or Petri Nets based service composition. Most of service composition techniques are automated and web based service composition in regards to its privileges in the comparisons with other techniques to enhance the achievement of composition objectives. Our future work is to assess the possibility and additionally those points of interest about the feasibility as well as the advantages and disadvantages of different service composition approaches.

\section{References}

Abbas, S., Rahamatullah, K., \& Paul, M. (2012). Template Based Composition for Requirements Based Network Stacks. IEEE, 2012. 978-4673-4410-4/12.

Al Khattab, A., Al-Shalabi, H., Al-Rawad, M., Al-Khattab, K., \& Hamad, F. (2015). The Effect of Trust and Risk Perception on Citizen's Intention to Adopt and Use E-Government Services in Jordan. Journal of Service Science and Management, 8(03), 279.

Alexis, A. A. P., Otavio, A. S. C., Bruno, G. B., Dionisio, M. L. F., Maycon, L. P., \& Bruno, T. K. (2017). Planning and Execution of Heterogeneous Service Compositions. International Conference on High Performance Computing \& Simulation, 2017.

Alexis, H., Ivan, S., \& Frank, S. (2007). Planning and Execution of Heterogeneous Service Compositions. IEEE, 2017. 978-1-5386-1629-1/17/\$31.00 @2017 IEEE.

Al-Sayyed, R. M., Fakhouri, H. N., Murad, S. F., \& Fakhouri, S. N. (2017). CACS: Cloud Environment Autonomic Computing System. Journal of Software Engineering and Applications, 10(03), 273.

Al-Sayyed, R. M., Fakhouri, H. N., Rodan, A., \& Pattinson, C. (2017). Polar Particle Swarm Algorithm for Solving Cloud Data Migration Optimization Problem. Modern Applied Science, 11(8), 98.

Baldin, S. H, \& Ilya. (2016). Dynamic Market-based Path Service Composition for Software Defined Networks. IEEE, 2016.

Bielikova, P. B., \& Maria. (2011). Automatic Dynamic Web service composition: A survey and problem formalization. Journal on Computing and Informatics, 30.

Dan, W., Bijan, P., Evren, S., James, H., \& Dana, Nau. (2013). Automating DAML-S Web Services Composition Using SHOP2. IEEE, 2013, International Conference on Advances in Computing, Communications and Informatics (ICACCI).

Danapaquiame, N., Ilavarasan, E., Muthumanickam, K., \& Poonkuzhali, N. (2015). A verification strategy for web services composition using enhanced stacked automata model. SpringerPlus, 2015.

Dong, L., Jiechu, Wu, Zehang, D., Zelin, Ch., \& Yang, Xu. (2017). QoS-Based Service Selection Method for Big Data Service Composition. IEEE, 2017, IEEE International Conference on Computational Science and Engineering (CSE) and IEEE International Conference on Embedded and Ubiquitous Computing (EUC).

Elli, R., Anthony, K., \& Vassilis, C. G. (2015). Decentralised Service Composition using Potential Fields in Internet of Things Applications. The 6th International Conference on Ambient Systems, Networks and Technologies (ANT 2015). Procedia Computer Science 52, 2015, ScienceDirect, ELSEVIER, pp. $700-$ 706. 
Hamad, F., \& Adwan, O. (2018). Policy Based Approach for Information Transfer over Mobile ad hoc Network using Messages Privacy Control. Modern Applied Science, 12(5), 22.

Hela, F., Sabri, M., \& Sadok, B. (2017). Local-Consistency Web Services Composition Approach Based on Harmony Search. Marseille, France: ELSEVIER, International Conference on Knowledge Based and Intelligent Information and Engineering Systems, September 6-8, 2017, pp. 1102-1111.

Hudaib, A. A., \& Fakhouri, H. N. (2016). An Automated Approach for Software Fault Detection and Recovery.

Hudaib, A. A., Fakhouri, H. N., Al Adwan, F. E., \& Fakhouri, S. N. (2016). A Survey about Self-Healing Systems (Desktop and Web Application). Communications and Network, 9(01), 71.

JARIR, Ilyass EL KASSMI \& Zahi. (2016). Security Requirements in Web Service Composition: Formalization, Integration and Verification. IEEE, 2016, 25th International Conference on Enabling Technologies: Infrastructure for Collaborative Enterprises (WETICE).

Jeyarani, R. A. (2012). E-Government application using service oriented architecture with an integration of SWORD. IEEE, 2012, International Conference on Emerging Trends in Science, Engineering and Technology (INCOSET).

Junjie, M., Hongming, C., \& Wei, W. (2016). A Web of Things Based Device-adaptive Service Composition Framework. IEEE, 2016, International Conference on e-Business Engineering.

Jyotishman, P., Samik, B., Robyn, L., \& Vasant, H. (2011). MoSCoE: A Framework for Modeling Web Service Composition and Execution. Computing and Informatics, 30, 793-827.

Kalamegam, P. (2017). Usage of CPN Models in Web Service Compositions. International Conference on Technical Advancements in Computers and Communications.

Kim, S. I. K., \& Hwa, S. (2016). Ontology-based Open API Composition Method for Automatic Mash-up Service Generation. IEEE, 2016.

Lifang, R., Wenjian, W., \& Hang, X. (2017). A Reinforcement Learning Method for Constraint-Satisfied Services Composition. IEEE, 14th International Conference on Services Computing, 2017. https://doi.org/10.1109/TSC.2017.2727050

Pal, A. D., \& Sanjukta. (2017). Dynamic Service Composition towards Database Virtualization for Efficient Data Management. IEEE, 2017. 978-1-5090-3519-9.

Rohit, A., Kunal, V., John, M., \& William, M. (2015). Constraint Driven Web Service Composition in METEOR-S. IEEE, 2015, IEEE Conference Publications.

Sowmya, V. S., Kamath, S., \& Ananthanarayana. (2013). A Bottom-up Approach Towards Achieving Semantic Web Services. IEEE, 2013, International Conference on Advances in Computing. 978-1-4673-6217-7/13.

Suda, Keita, F., \& Tatsuya. (2009). Semantics-based context-aware dynamic service composition. ACM, 2009, Trans. Auton. Adapt. Syst., 4.

Wang, Z., \& Wan, P. (2017). A Novel Pattern Based Dynamic Service Composition Framework. IEEE, 2017. 978-1-5090-3519-9.

Yuqian, L., \& Xun, X. (2017). A semantic web-based framework for service composition in a cloud manufacturing environment. ScienceDirect, ELSERIER, 2017. Journal of Manufacturing Systems, 42, 69-81.

\section{Copyrights}

Copyright for this article is retained by the author(s), with first publication rights granted to the journal.

This is an open-access article distributed under the terms and conditions of the Creative Commons Attribution license (http://creativecommons.org/licenses/by/4.0/). 